\title{
BAILLARGEON, Noël, Le Séminaire de Québec de 1685 à 1760. Québec, Les Presses de l'Université Laval, 1977. IX-459 p., 23 cm, index, cartes, plans et ill. (Les Cahiers d'histoire de l'Université Laval, no 21), \$18.00.
}

\section{Maurice Fleurent}

Volume 32, numéro 1, juin 1978

URI : https://id.erudit.org/iderudit/303674ar

DOI : https://doi.org/10.7202/303674ar

Aller au sommaire du numéro

Éditeur(s)

Institut d'histoire de l'Amérique française

ISSN

0035-2357 (imprimé)

1492-1383 (numérique)

Découvrir la revue

Citer ce compte rendu

Fleurent, M. (1978). Compte rendu de [BAILLARGEON, Noël, Le Séminaire de Québec de 1685 à 1760. Québec, Les Presses de l’Université Laval, 1977.

IX-459 p., $23 \mathrm{~cm}$, index, cartes, plans et ill. (Les Cahiers d'histoire de l'Université Laval, no 21), \$18.00.] Revue d'histoire de l'Amérique française, 32(1), 95-98. https://doi.org/10.7202/303674ar d'utilisation que vous pouvez consulter en ligne. 


\section{COMPTES RENDUS}

Baillargeon, Noël, Le Séminaire de Québec de 1685 à 1760, Québec, Les Presses de l'Université Laval, 1977, IX-459 p., $23 \mathrm{~cm}$., index, cartes, plans et ill. (Les Cahiers d'histoire de l'Université Laval, no 21), \$18.00.

L'ambitieux projet auquel Noël Baillargeon travaille depuis plus de dix ans, celui d'écrire l'histoire du Séminaire de Québec, se matérialise à un rythme qui autorise bien des espoirs. Avec cet ouvrage, qui fait suite à une remarquable étude parue en 1972 aux Presses de l'Université Laval, et intitulée Le Sóminaire de Québec sous l'épiscopat de Mgr de Laval, l'auteur complète l'histoire du Séminaire de Québec au temps de la Nouvelle-France.

L'ouvrage que Monsieur Baillargeon nous présente aujourd'hui est construit à partir de sources de première main. L'A. a dépouillé et passé au peigne fin une documentation énorme. Il s'est astreint à un contrôle rigoureux des faits au point de ne pas hésiter à se corriger lui-même (p. 355, note $15 ;$ p. 425 ). À cet égard, il se pourrait bien que l'ouvrage de Baillargeon ne vieillisse pas, et devienne un précieux instrument de travail pour tous les historiens et amateurs d'histoire intéressés aux institutions religieuses de la Nouvelle-France.

L'A. divise son ouvrage en deux parties de neuf chapitres chacune. Dans la première partie, il étudie principalement la qualité des rapports que Mgr de St-Vallier entretient avec le Séminaire de Québec. Ce qui en agacera plus d'un dans ce récit, fort bien mené par ailleurs, c'est la difficulté que paraît éprouver Baillargeon à cacher le préjugé assez défavorable qu'il donne l'impression d'entretenir envers Mgr de St-Vallier. Après avoir concédé que St-Vallier avait de la naissance et de la fortune, qu'il était bien vu à la Cour et qu'on lui reconnaissait beaucoup de piété, l'A. sert à son lecteur un euphémisme savoureux, et de facture essentiellement ecclésiastique, pourrait-on dire: "Monsieur de Saint-Vallier, naturellement, n'a pas que des qualités; il a bien aussi quelques défauts" (p. 6). Or, l'A. consacre ses neuf chapitres à démontrer que les principales difficultés que le Séminaire de Québec doit surmonter, de 1685 à 1727, sont dues aux «quelques défauts » de St-Vallier.

L'A. considère d'abord l'abbé de St-Vallier comme un personnage décevant, en qui Mgr de Laval et les co-fondateurs du Séminaire ont mis,

RHAF, vol. $32, \mathrm{n}^{\circ} 1$, juin 1978 
bien à tort, beaucoup d'espoir, et au sujet duquel, vu sa grande faveur à la Cour, ils se sont fait des joies fort prématurées. Maladroit et obstiné, assez peu sensible aux réprimandes de son prédécesseur qu'il écarte, un temps, de son chemin, Mgr de St-Vallier se révèle très tôt un homme de caractère autocratique. Rempli de préjugés, persistant dans ses préventions à l'égard du Séminaire, exigeant et intransigeant dans ses réclamations, accusateur, Mgr de St-Vallier est rempli d'aversion envers Mgr de Laval. De plus, «l'irréductible prélat» (p. 126) fait des promesses fallacieuses au Séminaire, mène une "politique contradictoire» (p. 128) à l'égard des curés, et agit «contre toute justice» (p. 128) dans l'affectation des fonds destinés aux curés, lors de l'établissement de l'Hôpital Général. L'A. écrit: «Mgr de Saint-Vallier n'avait que faire du droit et de la vérité quand il croyait que son autorité était en péril» (p. 145). Aussi, lors de ses démêlés avec le Chapitre, Mgr de Saint-Vallier émet-il une ordonnance qui n'est «qu'un tissu de faussetés» (p. 146). L'A. parle encore de la duplicité de Mgr de SaintVallier, et de sa manie habituelle d'user de représailles envers les directeurs du Séminaire de Québec, quand il n'est pas satisfait de leur comportement.

De l'avis de l'A., quand Mgr de St-Vallier travaille à détacher les cures et le Chapitre du Séminaire, et à réduire ce dernier à un rôle strictement éducatif et missionnaire, il cherche essentiellement à détruire l'œuvre de Mgr de Laval. L'A. ne discerne aucune raison pastorale valable dans l'action de St-Vallier. Il juge plutôt ce dernier à la façon, très négative, de Mgr de Laval. Il se représente St-Vallier, avant tout comme un homme disposé à employer, ainsi que l'écrivait Mgr de Laval, "tous les moyens que l'esprit humain et du démon peut former et inventer» (p. 144) pour arriver à ses fins. En voulant ainsi réduire l'empire que le Séminaire exerce sur son clergé, Mgr de St-Vallier, soutient l'A., renforce involontairement la dépendance du Séminaire de Québec de la maison mère de Paris, et indispose une partie de son clergé. D'autre part, l'invitation que le roi fait à Mgr de St-Vallier, à venir s'expliquer en France, est l'occasion d'une heureuse trève entre l'évêque et le Séminaire. Cependant, dès 1706, la guerre reprend de plus belle entre les deux parties, et l'évêque n'a de repos que lorsqu'il a réussi à dépouiller le Séminaire d'une grande partie de ses biens.

Dans la seconde partie de son ouvrage, Baillargeon s'attaque directement à l'histoire du Séminaire des Missions-Étrangères de Québec, à partir de la démission de Mgr de Laval, survenue en 1685, jusqu'à 1760 . Il consacre son premier chapitre à l'étude des règlements du Séminaire, de la formation de la jeunesse et de l'institution des domestiques «donnés». Les autres chapitres retracent l'histoire des entreprises de tous ordres mises sur pied par le séminaire, de 1685 à 1760 ; ils font en outre l'examen des crises financières et des principaux malheurs qui secouent l'institution durant la même période.

L'A. montre bien comment le Séminaire de Québec est amené, par l'acquisition de nombreux domaines, à se muer en grand constructeur, et 
par là, à faire des dépenses si fortes et à consentir des investissements si lourds que les Messieurs du Séminaire de Paris craignent pour la survie du Séminaire de Québec. Comme seigneur, le Séminaire doit en effet aménager les fermes de ses domaines, construire des manoirs, des moulins, des chapelles, des églises et des presbytères, des écoles et des couvents.

L'étude du «temporel» du Séminaire, comme on disait alors, amène l'A. à fournir un certain nombre de renseignements inédits sur les fonctions socio-économique et socio-culturelle exercées par le Séminaire au temps de la Nouvelle-France. L'A. renouvelle ainsi, en particulier, la compréhension du rôle joué par le Séminaire en agriculture et en éducation. Par exemple, le Séminaire de Québec paraît se situer à l'avant garde, dans l'utilisation des techniques agricoles nouvelles, tel l'assolement. D'autre part, le Séminaire prend une part très active en éducation. Dans ses domaines, il s'occupe avec autant d'ardeur de l'éducation des filles que de celle des garçons. Quant à la fameuse «école des arts et métiers de Saint-Joachim», l'A. nous en donne une réinterprétation (p. 232 à 234) qui devrait satisfaire, en partie du moins, le professeur Moogk ${ }^{1}$. Il faut souligner enfin la nouveauté et la qualité des deux derniers chapitres de l'ouvrage, portant sur les entreprises missionnaires du Séminaire en Acadie (1688-1732) et en Louisiane (1698-1768).

Au total, nous sommes en présence d'un excellent ouvrage, et fort bien écrit, qui comble une lacune grave de notre historiographie. La présentation typographique est à peu près parfaite, si l'on excepte la note 31 de la page 358 qui a échappé à l'impression. En outre, l'A. nous fournit un excellent index onomastique; il faut regretter, à cet égard, que l'A. n'ait pas jugé bon de nous donner un index thématique aussi parfait. Peut-être faut-il voir dans ce fait un indice du type de préoccupations qui ont guidé l'A. dans la construction de son ouvrage.

Les historiens seront sans doute tentés de réinterpréter un jour le rôle joué par les évêques de la Nouvelle-France, surtout par Mgr de St-Vallier, dans l'histoire du Séminaire de Québec, à la lumière des deux grandes forces qui s'affrontent toujours dans l'Église, la tendance à l'institutionalisation et le sens du renouveau. Ils seront probablement plus critiques aussi vis-à-vis des grandes orientations prises par le fondateur et maintenues si vigoureusement par les directeurs. Ils s'interrogeront sans doute avec plus de rigueur sur le refus de l'institution de canadianiser ses cadres supérieurs, en regard de la création rapide d'un clergé autochtone, et sur la portée socio-économique des grands domaines acquis par le Séminaire. Néanmoins,

1 Peter N. Moogk, «Réexamen de l'École des arts et métiers de SaintJoachim», RHAF, 29, 1 (juin 1975): 3-29. À ce sujet, voir aussi le commentaire que l'article de Moogk inspire à Lucien Campeau: " $A$ ' propos de l'École des arts et métiers de Saint-Joachim», ainsi que la réplique de Moogk à Campeau: "Réplique au commentaire du Père Campeau», dans RHAF, 29, 4 (mars 1976): 567-576. 
Noël Baillargeon a fait une œuvre extrêmement utile en nous livrant ce produit de qualité, même si quelques-unes de ses interprétations paraissent parfois se ressentir quelque peu de sa condition de fils spirituel du «Séminaire des Missions-Étrangères établi à Québec».

Département des Sciences humaines

Maurice Fleurent

Université du Québec à Trois-Rivières 